\title{
Analysis of Race and Gender Disparities in Incidence-Based Mortality in Patients Diagnosed with Thyroid Cancer from 2000 to 2016
}

This article was published in the following Dove Press journal: International Journal of General Medicine

\author{
Sunny Patel (D) \\ Lakshmi Pappoppula ${ }^{2}$ \\ Achuta Kumar Guddati (iD ${ }^{3}$ \\ Pavan Annamaraju ${ }^{4}$ \\ 'Department of Hematology/Oncology, \\ Medical College of Georgia, Augusta \\ University, Augusta, GA 30909, USA; \\ ${ }^{2}$ Division of Hematology/Oncology, \\ Georgia Cancer Center, Augusta \\ University, Augusta, GA 30909, USA; \\ ${ }^{3}$ Department of Hematology/Oncology, \\ Georgia Cancer Center, Augusta \\ University, Augusta, GA 30909, USA; \\ ${ }^{4}$ Johnston Memorial Hospital Ballad \\ Health System, Abingdon, VA \\ 242II, USA
}

Background: Well-differentiated thyroid cancer has better outcomes compared to undifferentiated/anaplastic thyroid cancer. The incidence of well-differentiated thyroid cancer is known to be more in women whereas it is approximately the same in both genders for anaplastic thyroid cancer. The variability of incidence-based mortality across gender in the context of race has not been studied. This study analyzes the rates of incidence-based mortality from the years 2000 to 2016 amongst both the genders in four racial groups.

Methods: The Surveillance, Epidemiology, and End Results (SEER) database was utilized to conduct a nation-wide analysis for the years 2000 to 2016. Incidence-based mortality for all stages of well-differentiated and undifferentiated thyroid cancer was queried and the results were grouped by race (Caucasian/White, African American/Black, American Indian/ Alaskan Native and Asian/Pacific Islander) and gender. All stages and ages were included in the analysis. Two sample $t$-test was used to determine statistically significant difference between various subgroups.

Results: Incidence-based mortality rates (per 100,000) for well-differentiated and undifferentiated thyroid cancer for all races and both the genders were calculated. The incidencebased mortality rates for both genders are approximately the same despite a 2-3:1 difference in incidence. Anaplastic thyroid cancer has a higher mortality rate in Caucasian and Asian/ Pacific Islander women compared to men despite an equal ratio in incidence. As expected, the mortality rates of anaplastic thyroid cancer were significantly higher compared to welldifferentiated cancer across all races and genders. Also, Asian/Pacific Islander women have a higher rate of mortality compared to both the genders of Caucasian and African American races.

Conclusion: Incidence-based mortality for anaplastic thyroid cancer is higher in women in all races whereas there is no difference in mortality between men and women for welldifferentiated thyroid cancer. This is divergent from the incidence ratios noted in these malignancies. In the context of increasing incidence of thyroid cancer for the past few decades, this data suggests that additional resources may be devoted to decreasing the disparity of mortality in this gender.

Keywords: thyroid cancer, incidence-based mortality, disparity, race, gender

\section{Introduction}

The incidence of thyroid cancer in the United States has dramatically increased over the last three decades. ${ }^{1}$ A retrospective analysis assessing the incidence of thyroid cancer from 1974 to 2013 estimated that the overall incidence has been increasing by an average of $3 \%$ annually. $^{2}$ The reason for this rise in cases remains unclear, but
Correspondence: Achuta Kumar Guddati Department of Hematology/Oncology, Georgia Cancer Center, Augusta University, Augusta, GA 30909, USA

$\mathrm{Tel}+$ I 312-404-8928

Email aguddati@augusta.edu
International Journal of General Medicine 2020:I3 I589-I594 
it may be partially attributed to the overdiagnosis and detection of papillary thyroid carcinoma - an indolent, differentiated form of thyroid cancer that rarely behaves aggressively. ${ }^{3,4}$ The increased utilization of imaging modalities like diagnostic ultrasound and biopsies has contributed to the incidental detection of these kinds of tumors which were unlikely to be found in the past without such sensitive diagnostic methods. ${ }^{5}$ As a result, thyroid cancer is now the most common endocrine malignancy with an estimated 52,890 cases in the United States. ${ }^{6}$

Unlike the consistent rise in thyroid cases, findings assessing mortality from thyroid cancer in the United States have been more variable. Multiple studies by Davies and colleagues have demonstrated a stable mortality rate of approximately 0.5 deaths per 100,000 patients for thyroid cancer from 1973 to $2009 .{ }^{3,7}$ In contrast, Lim et al found a $1.1 \%$ increase per year in mortality for patients with thyroid cancer from 1994 to $2013 .^{2}$ Yan et al also noted a similar increase in cancer-specific mortality in California from 2000 to $2017 .{ }^{8}$ Despite the differences, these studies still demonstrate a relatively low mortality rate in patients with thyroid cancer despite the rise in its incidence.

Thyroid cancer is classically categorized into the following distinct pathologic entities: well-differentiated thyroid cancer (which includes papillary and follicular thyroid cancers), medullary thyroid cancer, and anaplastic or poorly differentiated cancer. ${ }^{9}$ The grade of these tumors has been well recognized as a major prognostic factor in thyroid cancer. ${ }^{10}$ Patients with differentiated thyroid cancer have the best survival rates with an excellent 10-year survival ranging between 80 and $95 \%{ }^{11,12}$ Comparatively, patients with anaplastic tumors have a median survival of 5 months and less than $20 \%$ survive 1 year. ${ }^{13}$

Yet, these variances in survival do not only pertain to patients with different tumor grades. Cancer disparities are seen among gender and race as well. Aschebrook-Kilfoy et al assessed thyroid cancer incidence from 1992 to 2006 and found that whites have higher rates than other racial groups, which included blacks, Hispanics, and Asians. They further stratified their findings based upon gender and noted that Asian women have the highest incidence rate overall in the United States. ${ }^{14}$ This is one of the few studies that analyzed gender differences for thyroid cancer across racial groups. Other studies primarily focused upon gender disparities overall. As a result, it has been shown that thyroid cancer has a significant gender disparity for well-differentiated tumors with the incidence being approximately three-fold higher in women than men. ${ }^{15}$ Interestingly, men and women both present with similar incidence rates for more poorly differentiated forms of cancer. ${ }^{15}$ Some authors have even suggested that female gender could be an epidemiologic risk factor especially since no other risk factors, such as familial history or radiation exposure seem to account for the difference. $^{15,16}$ However, while female gender may be a risk factor for the increased rates of incidence, women are less likely to suffer mortality from thyroid cancer. In fact, men tend to have more aggressive disease at diagnosis and ultimately lower disease-free survival and higher mortality. ${ }^{17,18}$ Multiple studies have corroborated such disparities. ${ }^{16,19-21}$

We acknowledge that a modest amount of research regarding disparities in thyroid cancer has already been performed. As illustrated by the studies above, investigators have examined incidence and mortality trends of thyroid cancer with respect to various factors such as histologic grade, race, and gender in the United States. However, while comprehensive, no studies to our knowledge have analyzed mortality with respect to gender among different ethnicities on a national level. It remains unclear how mortality rates are affected among males and females stratified by their racial backgrounds. Thus, there is a need to assess the variability of incidence-based mortality with respect to gender and race over the past decade as such an assessment has not been conducted. To investigate the association between gender and race in thyroid cancer patients, we conducted a retrospective analysis using data from the Surveillance, Epidemiology, and End Results (SEER). This study aimed to evaluate and analyze the rates of disease-specific incidence-based mortality of male and female patients across various racial groups with well-differentiated and anaplastic thyroid cancer from the years 2000 to 2016 . We hope that this analysis may influence and ultimately improve thyroid cancer diagnosis and management for patients that may be at more risk.

\section{Methods}

\section{Data Source}

We utilized the SEER database of the National Cancer Institute's Surveillance Research Program in this retrospective cohort analysis to identify a population of patients with thyroid cancer. SEER collects data on cancer incidence, patient demographics, and tumor characteristics from 17 population-based cancer registries that cover approximately $34.6 \%$ of the United States population. 
The SEER areas include the states of Connecticut, Hawaii, Idaho, Iowa, Kentucky, Louisiana, Massachusetts, New Mexico, New York, Utah, Wisconsin; multi-county areas of Atlanta, rural Georgia, remaining counties of Georgia, San Francisco-Oakland, Seattle-Puget Sound, San JoseMonterey, Los Angeles county, remaining counties of California; and Arizona Indians, Alaska and Cherokee Nation. This study used deidentified data that is publicly available from the SEER database and thus approval by an institutional review board was not required.

\section{Study Population}

All patients diagnosed with thyroid cancer between 2000 and 2016 were eligible for the study. Patients were identified based on tumor site and histology using the World Health Organization's criteria in the third edition of the International Classification of Diseases for Oncology (ICD-O-3). The histologic codes of thyroid cancer were papillary carcinoma (8050, 8052, 8260, 8340-8341, 8343-8344, 8350), follicular carcinoma (8290, 8330-8332, 8335), medullary carcinoma (8345-8346, 8510, 8520), and anaplastic carcinoma (8012, 8020-8021, and 8030-8032). Patients of all ages with histologically confirmed well-differentiated and undifferentiated/ anaplastic thyroid tumors indiscriminate of clinical staging were included and categorized by race into 1 of 4 racial/ ethnic groups: Caucasian/white, African American/black, American Indian/Alaskan Native (AI/AN), and Asian/ Pacific Islander. We excluded patients from the study whose race and ethnicity were unknown or had a diagnosis of thyroid cancer associated with other malignancies or with unknown histology.

\section{Outcomes}

Age-adjusted thyroid cancer incidence-based mortality rates were calculated for gender, race/ethnicity, and tumor grade. All rates were adjusted to the 2000 US standard population and conveyed as the number of cases per 10,000 persons per year to limit confounding from different age distributions. Overall incidence-based mortality rates of thyroid cancer were defined as the number of deaths due to thyroid cancer among the total number of patient cases with the disease diagnosed over person-time at risk in SEER areas. ${ }^{22,23}$

\section{Statistical Analysis}

Incidence-based mortality was calculated using SEER*stat software. We used T-tests to compare mortality rates among both genders across the various racial subgroups listed above. $\mathrm{P}$-value $<0.05$ was considered statistically significant.

\section{Results}

Between the years of 2000 to 2016, a total of 3102 cases involved patients with a diagnosis of thyroid cancer. Incidence-based mortality rates (per 100,000) for welldifferentiated and anaplastic/undifferentiated thyroid cancer for both races and genders are shown in Table 1 .

The incidence-based mortality rates for both genders are approximately similar despite a 2-3:1 difference in incidence. Female patients tend to have higher rates of mortality than men when stratified by race and gender. Anaplastic thyroid cancer has a higher mortality rate in Caucasian and Asian/ Pacific Islander women compared to men despite an equal ratio of incidence. Women with only anaplastic thyroid cancer have a higher incidence-based mortality than their male counterparts. Asian/Pacific Islander women have a higher rate of mortality compared to both the genders of Caucasian and African American races. The mortality rates of anaplastic thyroid cancer were significantly higher compared to welldifferentiated cancer across all races and genders. Statistically significant differences are depicted in Table 1.

\section{Discussion}

The incidence of thyroid cancer has increased from 7.3 per 100,000 in 2000 to 14.1 per 100,000 in 2016 as per National cancer Institute statistics. This study was a population-based evaluation of thyroid cancer-specific incidence-based mortality rates in the United States utilizing the SEER database. We identified patients diagnosed with all stages of well-differentiated and anaplastic thyroid

Table I Incidence-Based Mortality Rates for Well-Differentiated and Anaplastic Thyroid Cancer Stratified by Race and Gender

\begin{tabular}{|c|c|c|c|}
\hline \multirow[t]{2}{*}{ Race } & \multirow[t]{2}{*}{ Gender } & \multicolumn{2}{|l|}{ Grade } \\
\hline & & Well-Differentiated & $\begin{array}{l}\text { Undifferentiated/ } \\
\text { Anaplastic }\end{array}$ \\
\hline \multirow[t]{2}{*}{ Caucasian/White } & Male & 0.0867 & 0.129 \\
\hline & Female & 0.0874 & $0.1429 *$ \\
\hline African & Male & 0.075 & 0.1015 \\
\hline American/Black & Female & 0.098 & $0.1286 *$ \\
\hline American Indian/ & Male & 0.0354 & $0.0724 *$ \\
\hline Alaskan Native & Female & $0.132 *$ & 0.0905 \\
\hline Asian/Pacific & Male & 0.0902 & 0.1257 \\
\hline Islander & Female & 0.1195 & $0.2033^{*}$ \\
\hline
\end{tabular}

Note: *Denotes statistical significance by $t$-test. 
cancer from 2000 to 2016 and assessed the variability in mortality among male and female patients across racial/ ethnic backgrounds. This study was the first national investigation to our knowledge to specifically evaluate these factors in this patient population.

The present analysis demonstrated that women with anaplastic thyroid cancer have a higher incidence-based mortality than their male counterparts while no significant difference in mortality was noted among genders for welldifferentiated forms of the malignancy. Female patients are known to experience higher rates of anaplastic thyroid cancer $^{24}$ but are generally associated with lower diseasespecific mortality. ${ }^{25}$ Despite previous evidence concluding the contrary, our finding that mortality rates in females are higher than males with anaplastic cancer is still very plausible since management against anaplastic thyroid cancer has been futile resulting in a disease that progresses rapidly with consistently low survival rates. ${ }^{24,26,27}$ Similar to our study, other investigations have shown comparable differences in mortality between genders as well. ${ }^{28}$ One ecological worldwide study in particular demonstrated that the mortality rate in females was 2.5 times higher than males and corroborated the female gender as a risk factor for thyroid cancer. ${ }^{28,29}$ However, it is important to note that these studies assessed thyroid cancer in general rather than specifically analyzing mortality rates among gender stratified by tumor grade.

Unlike the significant gender disparity noted with anaplastic thyroid cancer, our study did not find any difference in mortality among male and female patients with welldifferentiated tumors even though a recognized difference in incidence rates among the two genders exists. ${ }^{15}$ Analysis regarding gender disparities in populations with these types of tumors has always been controversial. Many earlier studies have found worse outcomes in men with differentiated thyroid cancer than women, ${ }^{17,20}$ while others have highlighted no significant difference in mortality among male and female patients. ${ }^{3,30}$ The findings of this study may be a reflection of refinements in histologic classification and staging of tumors as well as improvements in therapeutic management and surveillance ${ }^{31}-$ both of which have contributed to the high survival rates associated with differentiated tumors and thus reduced the influence of risk factors like gender on mortality.

In addition to the large gender disparity in incidencebased mortality noted among female patients with anaplastic thyroid cancer, it was interesting to find a similar difference in incidence-based mortality for well-differentiated tumors in the $\mathrm{AI} / \mathrm{AN}$ population. AI/AN women with welldifferentiated tumors had higher rates of mortality compared to their male counterparts. Small case counts have often limited accurate analysis of this population in the United States. $^{2,14}$ As a result, it was challenging to find many studies in the literature that specifically assessed this patient population. However, we were still able to draw conclusions based on the literature. Similar to our study, Nash et al illustrated that the AI/AN population is more likely to suffer from welldifferentiated thyroid malignancies, the two most common being papillary thyroid cancer and follicular thyroid cancer. These studies also found women to have a higher incidence of the disease compared to men. Moreover, compared to whites, most thyroid cancer tumors in this patient population were noted to be larger in size at initial diagnosis and regional in location rather than localized. ${ }^{32}$ Despite having welldifferentiated disease, the $\mathrm{AI} / \mathrm{AN}$ seem to have more advanced disease at diagnosis and thus are more likely to suffer mortality. This can also be further compounded by healthcare limitations and disparities. Many of these individuals live in remote areas making it difficult for them to access providers on a routine basis. The disparity is even worse for women. One study interviewed AI/AN women with a variety of cancers specifically and noted that they face obstacles in many areas, such as social injustices, medical autonomy, identifying their cancer, in accessing healthcare resources, and in personal interactions with certain providers who do visit areas. ${ }^{33}$ Women in this population not only face injustices outside of their vicinity but also with their own homes. Therefore, this is a very likely explanation for why we perceive higher rates of mortality in women compared to men for well-differentiated.

Finally, this study also found a higher rate of mortality in Asian women compared to all other genders and races. Our study is one of the few investigations that has assessed the differences in gender among ethnicities. The overall mortality rates noted in our analysis were lower than the findings of other studies. ${ }^{2,3,8}$ Interestingly, the trends among racial groups themselves were quite similar to Yan and colleagues who specifically studied incidence and mortality trends of thyroid cancer in California. They found mortality was highest in Asian patients at 0.39 per 100,000 person years followed by white patients at 0.36 per 100,000 person years. ${ }^{8}$ The difference in overall mortality rates may be explained by the fact that our investigation utilized a national database incorporating data from states and areas where access to health care be more readily available, allowing for better management of 
thyroid cancer. Our larger and thus higher-powered study likely provides a more accurate representation of thyroid cancer mortality on a national level. As for the respective differences in mortality rates among racial groups, it remains unclear why Asians with thyroid cancer have higher mortality rates. However, possible hypotheses do exist. One study proposed that Asians were more likely to have stage IV thyroid cancer with distant metastases. ${ }^{34}$ The presence of more advanced disease in this population could certainly account for the difference noted in mortality rates among Asian patients and patients of other racial groups seen in this study. Another explanation may involve the Filipino population. Filipinos account for the 2nd largest Asian population in the United States and may in fact be the most vulnerable to thyroid cancer. Nguyen et al demonstrated that Filipino women have the highest proportionate mortality rates compared to other racial/ ethnic/gender groups. Interestingly, rates were 3-5 times higher compared to white patients. ${ }^{35}$ Kus et al also demonstrated that Filipinos have a significantly increased risk of cancer recurrence compared to all other racial groups and thus may be more likely to suffer mortality attributed to thyroid cancer. ${ }^{36}$ Our study does not differentiate the Filipino population from the Asian population and thus this may provide a possible explanation for our results.

This study is not without limitations. We were limited by typical issues associated with the utilization of a registry database: incomplete data collection, inaccurate entry of information, and possible inconsistencies in tumor classification from changing perspectives on staging. Such concerns may have resulted in potential biases in our analysis. The utilization of SEER also limited our patient population to roughly one-third of the US population, which may not provide an accurate representation of mortality associated with all cases of thyroid cancer. This study also focused on racial discrepancies without assessing factors such as socioeconomic status and healthcare accessibility that could directly or indirectly affect thyroid cancer mortality rates over the years.

\section{Conclusion}

Using a national database, this study analyzes the rates of incidence-based mortality with respect to gender and race/ ethnicity of patients with well-differentiated and anaplastic thyroid cancer from the years 2000 to 2016. Our study underscores a higher rate of incidence-based mortality for anaplastic thyroid cancer in female patients of all racial backgrounds. No such differences in mortality were noted among male and female patients with well-differentiated thyroid cancer even though past studies have noted significant discrepancies in incidence among the two genders. We also found that Asian patients, particularly Asian women, have worse outcomes with higher mortality rates compared to other gender and racial groups. This retrospective analysis identifies individuals who are more likely to suffer mortality from thyroid cancer based on gender and race and warrants the allocation of clinical resources and monitoring for such populations to reduce cancer disparity.

\section{Funding}

The authors declare that there was no funding for this study.

\section{Disclosure}

All authors have read the manuscript and agree to the content. The authors report no conflicts of interest for this work.

\section{References}

1. Morris LG, Tuttle RM, Davies L. Changing trends in the incidence of thyroid cancer in the United States. JAMA Otolaryngol Head Neck Surgery. 2016;142(7):709-711. doi:10.1001/jamaoto.2016.0230

2. Lim H, Devesa SS, Sosa JA, Check D, Kitahara CM. Trends in thyroid cancer incidence and mortality in the United States, 1974-2013. JAMA. 2017;317(13):1338-1348. doi:10.1001/jama.2017.2719

3. Davies L, Welch HG. Current thyroid cancer trends in the United States. JAMA Otolaryngol Head Neck Surgery. 2014;140(4):317-322. doi:10.1001/jamaoto.2014.1

4. La Vecchia C, Malvezzi M, Bosetti C, et al. Thyroid cancer mortality and incidence: a global overview. Int $J$ Cancer. 2015;136 (9):2187-2195. doi:10.1002/ijc.29251

5. Sosa JA, Hanna JW, Robinson KA, Lanman RB. Increases in thyroid nodule fine-needle aspirations, operations, and diagnoses of thyroid cancer in the United States. Surgery. 2013;154(6):1420-1427. doi:10.1016/j.surg.2013.07.006

6. Siegel RL, Miller KD, Jemal A. Cancer statistics, 2020. CA Cancer J Clin. 2020;70(1):7-30.

7. Davies L, Welch HG. Increasing incidence of thyroid cancer in the United States, 1973-2002. JAMA. 2006;295(18):2164-2167. doi:10.1001/jama.295.18.2164

8. Yan KL, Li S, Tseng C-H, et al. Rising incidence and incidence-based mortality of thyroid cancer in California, 2000-2017. J Clin Endocrinol Metab. 2020;105(6):dgaa121. doi:10.1210/clinem/dgaa121

9. Carling T, Ocal IT, Udelsman R. Special variants of differentiated thyroid cancer: does it alter the extent of surgery versus well-differentiated thyroid cancer? World J Surg. 2007;31(5):916-923. doi:10.1007/s00268-006-0837-3

10. Shah JP, Loree TR, Dharker D, Strong EW, Begg C, Vlamis V. Prognostic factors in differentiated carcinoma of the thyroid gland. Am J Surgery. 1992;164(6):658-661. doi:10.1016/S0002-9610(05) 80729-9 
11. Schlumberger MJ. Papillary and follicular thyroid carcinoma. New England J Med. 1998;338(5):297-306. doi:10.1056/NEJM19980129338 0506

12. Sherma SI. Thyroid carcinoma. Lancet. 2003;361(9356):501-511. doi:10.1016/S0140-6736(03)12488-9

13. O'Neill JP, Shaha AR. Anaplastic thyroid cancer. Oral Oncol. 2013;49(7):702-706. doi:10.1016/j.oraloncology.2013.03.440

14. Aschebrook-Kilfoy B, Ward MH, Sabra MM, Devesa SS. Thyroid cancer incidence patterns in the United States by histologic type, 1992-2006. Thyroid. 2011;21(2):125-134. doi:10.1089/thy.2010. 0021

15. Rahbari R, Zhang L, Kebebew E. Thyroid cancer gender disparity. Future Oncol. 2010;6(11):1771-1779. doi:10.2217/fon.10.127

16. Yao R, Chiu CG, Strugnell SS, Gill S, Wiseman SM. Gender differences in thyroid cancer: a critical review. Expert Rev Endocrinol Metab. 2011;6(2):215-243. doi:10.1586/eem.11.9

17. Gilliland FD, Hunt WC, Morris DM, Key CR. Prognostic factors for thyroid carcinoma: A population-based study of 15,698 cases from the Surveillance, Epidemiology and End Results (SEER) Program 1973-1991. Cancer. 1997;79(3):564-573. doi:10.1002/(SICI)10970142(19970201)79:3<564::AID-CNCR20>3.0.CO;2-0

18. Kilfoy BA, Devesa SS, Ward MH, et al. Gender is an age-specific effect modifier for papillary cancers of the thyroid gland. Cancer Epidemiol Prevention Biomarkers. 2009;18(4):1092-1100. doi:10. 1158/1055-9965.EPI-08-0976

19. Jonklaas J, Nogueras-Gonzalez G, Munsell M, et al. The impact of age and gender on papillary thyroid cancer survival. J Clin Endocrinol Metab. 2012;97(6):E878-E887.

20. Machens A, Hauptmann S, Dralle H. Disparities between male and female patients with thyroid cancers: sex difference or gender divide? Clin Endocrinol. 2006;65(4):500-505. doi:10.1111/j.1365-2265. 2006.02623.x

21. Micheli A, Ciampichini R, Oberaigner W, et al. The advantage of women in cancer survival: an analysis of EUROCARE-4 data. Eur $J$ Cancer. 2009;45(6):1017-1027. doi:10.1016/j.ejca.2008.11.008

22. Chu KC, Miller BA, Feuer EJ, Hankey BF. A method for partitioning cancer mortality trends by factors associated with diagnosis: an application to female breast cancer. J Clin Epidemiol. 1994;47 (12):1451-1461. doi:10.1016/0895-4356(94)90089-2

23. Saad AM, Gad MM, Al-Husseini MJ, Ruhban IA, Sonbol MB, Ho TH. Trends in renal-cell carcinoma incidence and mortality in the United States in the last 2 decades: a SEER-based study. Clin Genitourin Cancer. 2019;17(1):46-57. e45. doi:10.1016/j. clgc.2018.10.002
24. Janz TA, Neskey DM, Nguyen SA, Lentsch EJ. Is the incidence of anaplastic thyroid cancer increasing: A population based epidemiology study. World j Otorhinolaryngol Head Neck Surgery. 2019;5 (1):34-40. doi:10.1016/j.wjorl.2018.05.006

25. Kebebew E, Greenspan FS, Clark OH, Woeber KA, McMillan A. Anaplastic thyroid carcinoma: treatment outcome and prognostic factors. Cancer. 2005;103(7):1330-1335. doi:10.1002/cncr.20936

26. Ranganath R, Shah MA, Shah AR. Anaplastic thyroid cancer. Curr Opin Endocrinol Diabetes Obes. 2015;22(5):387-391. doi:10.1097/ MED.0000000000000189

27. Smallridge RC, Copland J. Anaplastic thyroid carcinoma: pathogenesis and emerging therapies. Clin Oncol. 2010;22(6):486-497. doi:10.1016/j.clon.2010.03.013

28. Soheylizad M, Khazaei S, Jenabi E, Delpisheh A, Veisani Y. The relationship between human development index and its components with thyroid cancer incidence and mortality: using the decomposition approach. Int J Endocrinol Metabolism. 2018;16:4.

29. Pellegriti G, Frasca F, Regalbuto C, Squatrito S, Vigneri R. Worldwide increasing incidence of thyroid cancer: update on epidemiology and risk factors. J Cancer Epidemiol. 2013;2013.

30. Siironen P, Louhimo J, Nordling S, et al. Prognostic factors in papillary thyroid cancer: an evaluation of 601 consecutive patients. Tumor Biol. 2005;26(2):57-64. doi:10.1159/000085586

31. Lamartina L, Grani G, Durante C, Filetti S. Recent advances in managing differentiated thyroid cancer. F1000Research. 2018;7.

32. Nash SH, Lanier AP, Southworth MB. Occurrence of endocrine and thyroid cancers among alaska native people, 1969-2013. Thyroid. 2018;28(4):481-487. doi:10.1089/thy.2017.0408

33. Liddell JL, Burnette CE, Roh S, Lee Y-S. Healthcare barriers and supports for American Indian women with cancer. Soc Work Health Care. 2018;57(8):656-673. doi:10.1080/00981389.2018.1474837

34. Moo-Young TA, Panergo J, Wang CE, et al. Variations in clinicopathologic characteristics of thyroid cancer among racial ethnic groups: analysis of a large public city hospital and the SEER database. Am J Surgery. 2013;206(5):632-640. doi:10.1016/j.amjsurg.2013.07.015

35. Nguyen MLT, Hu J, Hastings KG, et al. Thyroid cancer mortality is higher in Filipinos in the United States: an analysis using national mortality records from 2003 through 2012. Cancer. 2017;123 (24):4860-4867. doi:10.1002/cncr.30958

36. Kus LH, Shah M, Eski S, Walfish PG, Freeman JL. Thyroid cancer outcomes in Filipino patients. Archives Otolaryngol Head Neck Surgery. 2010;136(2):138-142. doi:10.1001/archoto.2009.206
International Journal of General Medicine

\section{Publish your work in this journal}

The International Journal of General Medicine is an international, peer-reviewed open-access journal that focuses on general and internal medicine, pathogenesis, epidemiology, diagnosis, monitoring and treatment protocols. The journal is characterized by the rapid reporting of reviews, original research and clinical studies across all disease areas. The manuscript management system is completely online and includes a very quick and fair peer-review system, which is all easy to use. Visit http://www.dovepress.com/ testimonials.php to read real quotes from published authors. 